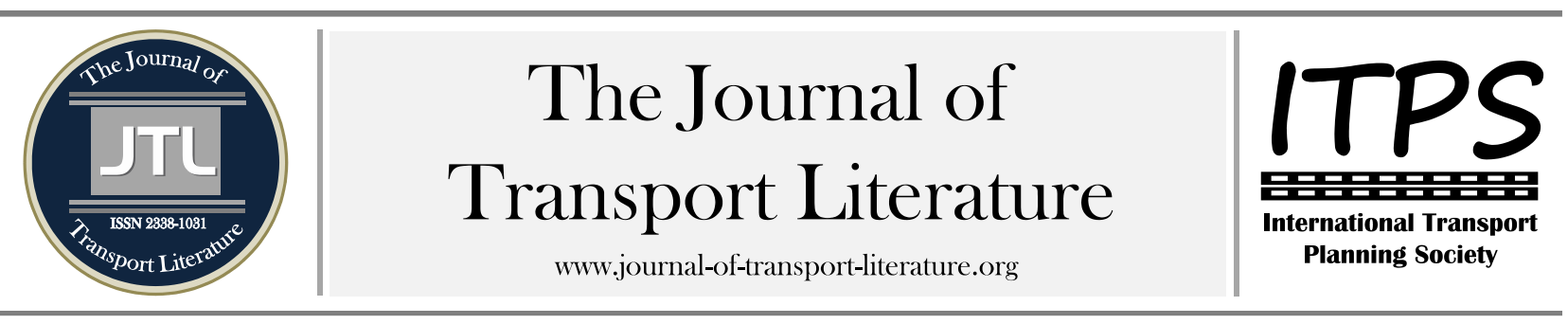

\title{
Uma meta-heurística de busca decomposta em vizinhança variável para o problema bidimensional de agrupamento de entregas em veículos de uma frota heterogênea
}

\author{
Jorge Von Atzingen dos Reis ${ }^{1,+}$; Claudio Barbieri da Cunha ${ }^{2}$ \\ 1 Federal University of Uberlândia, Ituiutaba, Brazil \\ 2 University of São Paulo, São Paulo, Brazil
}

\section{Article Info}

Palavras-chave:

busca decomposta em

vizinhança variável

problema bidimensional de

agrupamento e alocação de

entregas

meta-heurística

Submitted 27 Feb 2014;

received in revised form 5 Apr 2014;

accepted 8 Oct 2014.

Licensed under

Creative Commons

CC-BY 3.0 BR.

\section{Resumo}

Este artigo trata do problema de distribuição física que envolve o agrupamento e alocação de entregas a uma frota de veículos visando minimizar o frete total. Este problema surge em diferentes situações práticas em que os pontos a serem atendidos estão suficientemente próximos entre si de tal modo que as distâncias percorridas entre paradas consecutivas podem ser consideradas irrelevantes para o custo total de entregas da frota e, portanto, podem ser desconsideradas para o cálculo dos fretes pagos a terceiros que realizam esse serviço. Este trabalho propõe um método de resolução para um problema de binpacking generalizado com restrições adicionais encontrado em situações práticas de logística e transporte de cargas. Neste trabalho é proposta uma meta-heurística de Busca Decomposta em Vizinhança Variável (VNDS) para a resolução desse problema. Experimentos computacionais com instâncias de problemas benchmarking da literatura evidenciam que essa meta-heurística produz bons resultados (obteve a solução ótima ou próxima do limite inferior em todas as instâncias testadas) em tempos de processamento bastante reduzidos (até $5,10 \%$ mais rápido do que o VNS).

+ Corresponding author. Universidade Federal de Uberlândia, Faculdade de Ciências Integradas do Pontal. Rua Vinte, 1600. $38304-402$ Ituiutaba, MG - Brasil. E-mail address: orgereis@pontal.ufu.br.

\section{Introdução}

Neste trabalho é abordado o problema de bin-packing bidimensional com bins de tamanho variável (do inglês bidimensional variable sized bin-packing problem - 2-VSBPP). A principal diferença em relação ao VSBPP é a existência de uma segunda dimensão no problema, no 2-VSBPP considera-se que cada objeto possui um peso e um volume, assim como cada bin (caminhão) possui uma capacidade de carga e uma capacidade volumétrica. A contribuição deste trabalho é propor um método de resolução para um problema de bin-packing generalizado com restrições adicionais encontrado em situações práticas de logística e transporte de cargas. Outra contribuição deste trabalho é propor uma meta-heurística de Busca Decomposta em Vizinhança Variável (VNDS) para a resolução desse problema. O objetivo principal deste trabalho é testar um método de resolução baseado na Busca Decomposta em Vizinhança Variável (VNDS) utilizando dados de instâncias benchmarking da literatura e comparando os resultados com a solução ótima de algumas instâncias obtidas através da resolução de um modelo matemático.

A frota brasileira de veículos automotores cresceu 355\% entre 1990 e 2010 (de 18 para 64 milhões) apresentando um padrão não sustentável de evolução. Os transportes em geral consomem cerca de $28 \%$ da energia total do Brasil, sendo o transporte de carga responsável por 92\% deste consumo (Castro Júnior, 2013). Segundo Castro Júnior (2013), as crescentes demandas da sociedade por transporte tem desafiado a questão do desenvolvimento sustentável. 0 setor de transporte é um grande consumidor de recursos não renováveis e o esgotamento destes recursos já vem sendo anunciado, desta forma a otimização da utilização dos recursos é benéfica para toda a sociedade possibilitando o atendimento do anseio por transporte e mobilidade. No contexto logístico, a distribuição física de produtos geralmente envolve o roteamento de veículos, tendo em vista que o tamanho médio das cargas a serem entregues aos clientes, particularmente no meio urbano, não é suficiente para lotar um veículo, o que acarreta que cada veículo atenda a diversos clientes; consequentemente, buscase determinar quais entregas devem ser alocadas a cada veículo de uma frota disponível, e qual a sequência de paradas (ou roteiro) de cada veículo, de forma a minimizar o custo total do serviço, geralmente composto da soma ponderada dos custos proporcionais às distâncias percorridas e dos custos fixos dos veículos. Entretanto, em diversas situações práticas um problema diferente pode surgir, que envolve o transporte de várias cargas para um único destino, ou para vários destinos próximos entre si, quando se torna necessário otimizar o agrupamento de entregas nos veículos de modo a reduzir a frota necessária e o custo com o frete pago aos transportadores. Em muitos problemas pode-se assumir produto único ou homogêneo, como é o caso, por exemplo, do cimento ensacado (Miura, 2008), ou de produtos em caixas, ou paletes, ou até mesmo em gaiolas, permitindo assim desconsiderar a questão do arranjo espacial ou acomodação das cargas nos veículos. 
Como se trata de um problema de entregas de carga fracionada pode-se assumir, sem perda de generalidade, que nenhuma entrega supera a capacidade do menor veículo disponível.

Esse problema de distribuição pode ser modelado como um problema conhecido na literatura como o problema de binpacking (BPP), definido da seguinte forma: dado um conjunto de objetos (ou itens) com seus respectivos pesos e um conjunto de bins (ou mochilas) idênticas de capacidade finita, determinar a alocação (ou designação) dos itens aos bins, de tal modo que o número de bins utilizados seja mínimo, e a capacidade de cada bin não seja violada. No caso do problema de distribuição em questão, os veículos de uma frota homogênea correspondem aos bins, e os objetos (ou itens) às cargas a serem entregues, cujos respectivos clientes estão localizados em uma mesma localidade, ou em localidades próximas de tal sorte que frete de entrega não seja afetado pelas distâncias para atendimento das diferentes paradas para entrega ao longo do trajeto. No caso mais geral, a frota pode ser composta por veículos de diferentes tamanhos e capacidades; contendo dois ou mais tipos de veículos, aos quais estão associados custos diferentes. Nesse caso, busca-se não mais minimizar o número total de veículos, mas sim o custo (ou frete) total dos veículos utilizados. Esse problema mais geral com frota heterogênea é conhecido na literatura como o problema de bin-packing com bins heterogêneos, ou de tamanho variável (do inglês variable sized bin-packing problem, ou simplesmente VSBPP). Usualmente os veículos maiores possuem fretes unitários (por unidade de capacidade) menores, uma vez que tanto o custo fixo quanto o custo variável não são diretamente proporcionais à capacidade de carga do veículo; por exemplo, o consumo unitário de combustível não varia diretamente com a capacidade de carga; tampouco o salário do motorista costuma variar com o tamanho do veículo, o que faz com que, por exemplo, um veículo com o dobro da capacidade de carga do outro não tenha o seu custo fixo dobrado. No modal rodoviário o custo fixo costuma ser baixo em relação ao custo variável, o qual é elevado juntamente com o custo ambiental (Eller et al., 2011). Assim, tendo em vista a resolução de problemas reais de distribuição física de produtos, é proposta, neste trabalho, uma meta-heurística de Busca Decomposta em Vizinhança Variável (VNDS) para o problema 2-VSBPP.

Este artigo está organizado da seguinte forma: na próxima seção é apresentada uma breve revisão bibliográfica sobre o problema de bin-packing; já a seção 2 contém a modelagem matemática do problema. Em seguida, na seção 3 é descrita a meta-heurística de Busca Decomposta em Vizinhança Variável (Variable Decomposition Neighborhood Search - VNDS). Os resultados dos experimentos computacionais realizados são apresentados na seção 4, incluindo uma comparação dos resultados com os obtidos através de um pacote de otimização e com os resultados obtidos pela meta-heurística VNS utilizada por Reis \& Cunha (2010) para resolver o 2-VSBPP. Por fim, as conclusões e recomendações são apresentadas.

\section{0 problema de bin-packing com frota heterogênea}

Na literatura existem diversas heurísticas propostas para a resolução do Problema de Bin-packing (BPP). As mais populares são as heurísticas nas quais os objetos são ordenados em ordem decrescente de peso (Wj) e são aplicadas regras para a alocação dos objetos aos bins, tais como first-fit, best-fit ou next-fit (Coffman et al., 1997). Entretanto, no caso do problema de bin-packing com frota heterogênea (VSBPP), a literatura é bem mais restrita, e só mais recentemente esta generalização do problema clássico passou a atrair a atenção dos pesquisadores. O VSBPP é NP-hard, uma vez que se reduz ao BPP no caso particular em que todos os bins são idênticos, que é NP-hard (Garey e Johnson, 1979). Segundo Zhang (1997) as heurísticas clássicas para o BPP não são capazes de fornecerem bons resultados para o VSBPP. O trabalho de Friesen e Langston (1986) é um dos pioneiros sobre o VSBPP definindo limitantes (bounds) e apontando a direção de pesquisas futuras com a utilização de heurísticas. Burkard e Zhang (1997) e Kang e Park (2003) propõem algoritmos aplicados ao problema de VSBPP e determinam o desempenho no pior caso dos algoritmos para casos específicos. Kang e Park (2003) propõem dois algoritmos gulosos, os quais são testados em três casos especiais: os tamanhos dos itens e dos bins são divisíveis; somente o tamanho dos bins é divisível; ou o tamanho dos bins não é divisível.

Nos casos em que o tamanho dos itens e dos bins são divisíveis, o algoritmo guloso proposto obtém a solução ótima. Correia et al. (2008) utilizam uma formulação de programação linear inteira e uma reformulação do modelo de otimização discreta para a solução do VSBPP. Novas desigualdades sugeridas pelas variáveis do modelo discreto são adicionadas a fim de melhorar a relaxação dos limites do modelo linear original. Os resultados obtidos indicam que o modelo proposto obteve bons limites para a programação linear tornando-o adequado para resolver instâncias consideradas pequenas ou com até 1.000 itens. Para instâncias maiores o modelo ainda pode ser utilizado para a obtenção dos limites inferiores da solução. Mais recentemente, Haouari e Serairi (2009) analisaram o desempenho empírico de seis heurísticas, sendo quatro baseadas em métodos construtivos a partir da solução exata de subconjuntos dos dados; uma heurística baseada em Algoritmo Genético e uma heurística baseada em cobertura de conjuntos (set covering). As heurísticas propostas foram testadas e comparadas em conjuntos de dados, com até 2.000 objetos e sete tamanhos de bins, randomicamente gerados. A heurística baseada em cobertura de conjunto obteve sucesso conseguindo soluções de alta qualidade em baixo tempo computacional. Reis e Cunha (2011) resolvem o problema unidimensional de bin-packing com frota heterogênea utilizando uma metaheurística VNS e para a sua avaliação foram consideradas instâncias de teste derivadas de instâncias benchmarking da literatura para o BPP, adaptadas para o VSBPP. Também foi realizada uma comparação com os resultados exatos obtidos através da utilização de um pacote de otimização linear. Os resultados obtidos indicam que o algoritmo proposto permitiu obter a solução ótima na maioria dos testes e nos demais testes a solução obtida sempre esteve próxima do limitante inferior, sendo que as soluções ótimas encontradas encontram-se sempre acima dos respectivos limitantes, o que indica que a estratégia de solução proposta atinge resultados de excelente qualidade com tempos de processamento muito reduzidos.

\section{Modelagem matemática}

0 problema de bin-packing bidimensional com bins heterogêneos (2-VSBPP) pode ser descrito da seguinte forma: dados $j=1, \ldots, n$ objetos ou itens com seus respectivos pesos $w_{j}^{1}$ e volumes $w_{j}^{2}$, e $i=1, \ldots, m$ bins (ou mochilas) para os quais são conhecidos seus respectivos custos $c_{i}$ e suas capacidades em peso $b_{i}^{1}$ e volumétrica $b_{i}^{2}$, determinar a alocação (ou designação) dos $n$ objetos aos $m$ bins, de tal modo a minimizar o custo total dos bins utilizados, e respeitando-se as restrições de capacidade nos bins. Assume-se, sem perda de generalidade, que todos os objetos possuam um tamanho inferior à capacidade do menor bin e que o número de bins $m$ seja suficientemente elevado a fim de assegurar a viabilidade do problema. Definem-se ainda as seguintes variáveis de decisão: $x_{i j}$ é uma variável binária que assume o valor 1 quando o 
objeto $j$ é alocado ao bin $i$ e recebe o valor zero caso contrário; $y_{i}$ é uma variável binária que recebe o valor 1 quando o bin $i$ é utilizado na solução e assume o valor zero caso contrário. Assim, a formulação matemática do 2-VSBPP pode ser escrita da seguinte forma:

$$
\begin{gathered}
\operatorname{Min} \sum_{i=1}^{m} c_{i} y_{i} \\
\sum_{j=1}^{n} w_{j}^{1} x_{i j} \leq b_{i}^{1} y_{i} \quad \forall i=1, \ldots, m \\
\sum_{j=1}^{n} w_{j}^{2} x_{i j} \leq b_{i}^{2} y_{i} \quad \forall i=1, \ldots, m \\
\sum_{i=1}^{m} x_{i j}=1 \quad \forall j=1, \ldots, n \\
x_{i j} \in[0,1] \quad \forall i=1, \ldots, m \quad \forall j=1, \ldots, n \\
y_{i} \in[0,1] \quad \forall i=1, \ldots, m
\end{gathered}
$$

Onde: a função objetivo (1) busca minimizar o custo total dos veículos (ou bins) utilizados; As restrições (2) asseguram que a capacidade (peso) de cada veículo utilizado não seja violada; As restrições (3) garantem que a capacidade volumétrica de cada veículo utilizado não seja violada; As restrições (4) impõem que cada entrega (ou objeto) $j$ seja alocada a exatamente um veículo; As restrições (5) e (6) asseguram a integralidade das variáveis de decisão.

\section{Meta-heurística VNDS aplicada ao 2-VSBPP}

A resolução de um problema de bin-packing generalizado com restrições adicionais, encontrado em situações práticas de logística e transporte de cargas, através da utilização de um modelo matemático é limitada a instâncias de pequeno porte devido à natureza combinatória do 2-VSBPP (Reis e Cunha, 2011; Garey e Johnson, 1979). Uma forma de contornar a limitação dos pacotes de otimização para resolver modelos matemáticos para problemas da classe NP-hard é a utilização de meta-heurísticas. Segundo Glover e Kochenberg (2003), o VNS (Variable Neighborhood Search) é uma evolução do VND (Variable Neighborhood Descent) e possui similaridades com o ILS (Iterated Local Search). O VND, proposto em Mladenovic e Hansen (1997), é um método de busca local que explora o espaço de soluções por meio de trocas de estruturas de vizinhança aceitando somente soluções de melhora da solução corrente e retornando à primeira estrutura quando uma solução melhor é encontrada. Caso não seja encontrada uma solução de melhora a busca continuará na estrutura de vizinhança seguinte. Caso não exista uma estrutura de vizinhança seguinte a execução é interrompida e a solução corrente é retornada como a solução ótima local.

O VNS (Método de Pesquisa em Vizinhança Variável) é um método de busca local, o qual consiste em explorar o espaço de soluções, por meio de trocas sistemáticas de estruturas de vizinhança. Contrariamente a outras meta-heurísticas, baseadas em métodos de busca local, o método VNS não segue uma trajetória, mas sim explora vizinhanças gradativamente mais "distantes" da solução corrente e focaliza a busca em torno de uma nova solução se, e somente se, um movimento de melhora é realizado (Glover e Kochenberg, 2003). 0 método inclui, também, um procedimento de busca local a ser aplicado sobre a solução corrente. Esta rotina de busca local também pode usar diferentes estruturas de vizinhança. A metaheurística VNS explora o espaço de soluções a partir de uma solução corrente $s$ realizando um movimento de exploração da vizinhança e uma solução vizinha $s^{\prime}$ é selecionada dentro do raio de alcance da estrutura de vizinhança $N^{1}(s)$. Este movimento de exploração consiste em fazer uma modificação na solução corrente utilizando um critério de escolha aleatório para possibilitar que a meta-heurística VNS escape de ótimos locais. A partir desta solução vizinha $s^{\prime}$ é realizada uma busca local na vizinhança da solução $s^{\prime}$. Caso a melhor solução vizinha de $s^{\prime}$ não resulte em melhoria retorna-se à solução $s$. Uma solução $s^{\prime}$ será considerada de melhoria se $f\left(s^{\prime}\right)<f(s)$ (em um problema de minimização). Após retornar à solução corrente $s$ é realizado um novo movimento de exploração da vizinhança, escolhendo-se uma solução vizinha $s^{\prime \prime}$ pertencente ao raio de alcance da estrutura de vizinhança $N^{2}(s)$. Este movimento de exploração consiste em fazer uma modificação na solução corrente utilizando um critério de escolha aleatório para possibilitar que a meta-heurística VNS escape de ótimos locais. Neste momento é realizado uma busca local na vizinhança da solução $s^{\prime \prime}$. Caso a melhor solução vizinha de $s^{\prime \prime}$ seja aceita como solução de melhora, a partir dessa solução é escolhida uma solução $s^{\prime \prime \prime}$, dentro do raio de alcance da estrutura $N^{1}\left(s^{\prime \prime}\right)$ após a realização de um movimento de exploração da vizinhança. Se a solução $s^{\prime \prime \prime}$ for aceita uma nova solução $s^{\prime \prime \prime \prime}$, pertencente à vizinhança de $s^{\prime \prime \prime}$, é escolhida. Sempre que uma solução de melhora for encontrada pela meta-heurística VNS a solução corrente é movida para a nova solução e a busca é reiniciada considerando a primeira estrutura de vizinhança $N^{1}$. E na meta-heurística VNS a busca local sempre é realizada na vizinhança de uma solução vizinha (escolhida aleatoriamente) da solução corrente. Hansen et al. (2001) propuseram a meta-heurística VNDS (Método de Busca Decomposta em Vizinhança Variável) como uma variação da meta-heurística VNS proposta por Mladenovic e Hansen (1997). A principal mudança em relação ao VNS é a busca local que é decomposta em duas etapas.

0 VNDS funciona da seguinte forma: após ser gerada uma solução $s^{\prime}$ aleatória dentro da estrutura de vizinhança $k$, a primeira etapa da busca local é uma busca realizada na vizinhança de $s^{\prime}$ e utilizando a estrutura de vizinhança $k$. A segunda etapa da busca local é uma busca realizada dentro da vizinhança de $s^{\prime}$ e além da estrutura de vizinhança $k$ que está sendo analisada. Esta etapa ocorre quando não existe mais a possibilidade de se encontrar reinserções viáveis das entregas $j^{*}$ ao veículo $i^{*}$ para outro veículo $i$ que possua capacidade ociosa para receber esta entrega. Neste caso, é testado a troca de entregas entre o veículo $i^{*}$ e outros veículos $i$. Desta forma, esta segunda etapa do procedimento de busca local decomposta tenta redistribuir as entregas visando obter uma melhor alocação e a liberação de espaço sem aumentar o número de veículos necessários. 


\section{Experimentos Computacionais}

A meta-heurística VNDS implementada para resolver o 2-VSBPP foi testada com dados derivados de instâncias da literatura, mais especificamente as instâncias benchmarking do BPP disponíveis na OR Library, originalmente propostas por Falkenauer (1996) para o BPP, no qual os bins são idênticos, e que haviam sido utilizadas por Cunha et al. (2008). A principal diferença das novas instâncias geradas foi a consideração de mais dois tamanhos de bins (veículos) além daquele de capacidade igual a 150. Tomando por base o tamanho de bin correspondente à capacidade igual a 150 conforme proposto por Falkenauer (1996), e atribuindo um custo de 100, foram criados mais dois tipos de bins da seguinte forma: um tipo de bin com capacidade $25 \%$ inferior e custo $20 \%$ inferior e um tipo de bin com capacidade $25 \%$ superior e custo $20 \%$ superior, como apresentado na Tabela 1. Dessa forma, o bin de maior tamanho é o que apresenta o menor custo por unidade de capacidade, tal como ocorre na prática onde veículos maiores são os de menor custo unitário.

A diferença básica das novas instâncias foi a geração de uma capacidade volumétrica idêntica à capacidade em quilogramas do veículo e a adição de um volume para cada objeto a ser alocado aos bins. Por simplificação, considerou-se que as capacidades volumétricas dos bins são iguais às capacidades em peso. Entretanto, o peso $w_{j}^{1}$ e o volume $w_{j}^{2}$ de cada entrega $j$ diferem com o objetivo de configurar produtos com diferentes densidades de carga (ou, similarmente, fatores de estiva) de modo que os veículos possam lotar ou por peso ou por volume, dependendo das entregas alocadas a cada um deles. Para determinar o volume $w_{j}^{2}$ de cada entrega $j$ foi escolhido, aleatoriamente, um valor de volume correspondente ao intervalo entre $50 \%$ e $150 \%$ do valor do peso $w_{j}^{1}$ de cada entrega.

Tabela 1 - Custo e capacidade dos veículos. Fonte: Autoria própria.

\begin{tabular}{lccc}
\hline Dimensão & Grande & Médio & Pequeno \\
\hline Custo $(\$)$ & 120 & 100 & 80 \\
Capacidade $(\mathrm{kg})$ & 187 & 150 & 112 \\
Capacidade $\left(\mathrm{m}^{3}\right)$ & 187 & 150 & 112 \\
\hline
\end{tabular}

A função objetivo é utilizada para avaliar a qualidade de uma solução vizinha obtida através de um movimento realizado por uma das estruturas de vizinhança utilizadas pelas meta-heurísticas VNS ou VNDS. A função proposta visa a avaliar a qualidade de uma solução para o 2-VSBPP considerando o custo da frota alocada, sendo calculada através da Expressão (7).

$$
F O=\sum_{i=1}^{3} \text { Custo }_{i} \text { NumBinTipo }_{i}
$$

Onde: Custo $_{i}$ é o custo por utilizar um veículo do tipo $i$; NumBinTipo $i$ é a quantidade de veículos (bins) do tipo $i$ utilizados. Os testes foram realizados em um total de quatro conjuntos de dados com problemas com o número de objetos variando entre 120 e 1.000. As heurísticas propostas VNS e VNDS foram codificadas em C++. Tendo em vista a aleatoriedade dos métodos propostos, cada instância foi resolvida cinco vezes e as melhores soluções obtidas para cada instância estão apresentada na Tabela 2. A primeira coluna da Tabela 2 apresenta o limite inferior (lower bound) para cada uma das instâncias de teste. 0 limite inferior representa o custo mínimo para cada instância e é calculado considerando que todas as cargas sejam alocadas ao veículo com a melhor relação custo/benefício e este limite pode ser obtido pela Expressão (8).

$$
\text { LowerBound }=\frac{\text { Custo }_{G D E} \sum_{i=1}^{n} \text { Peso }_{i}}{\text { Capacidade }_{G D E}}
$$

Onde: Custo $_{G D E}$ é o custo por utilizar um veículo do tipo grande; Capacidade ${ }_{G D E}$ é a capacidade dos veículos do tipo grande; $\mathrm{Peso}_{i}$ é o peso do objeto $i$; $n$ é o total de objetos da instância do problema. A coluna FO da Tabela 2 apresenta o custo da solução obtida para cada instância pelo pacote de otimização Gurobi@ (o qual não possibilitou determinar a solução ótima em nenhuma das instâncias testadas) e pelas duas meta-heurísticas. A coluna Tempo apresenta o tempo computacional, medido em segundos, para a obtenção da solução apresentada por cada uma das meta-heurísticas implementadas.

Tabela 2 - Resultados obtidos.

\begin{tabular}{|c|c|c|c|c|c|c|c|}
\hline \multirow{2}{*}{$\begin{array}{l}\text { Número } \\
\text { de Objetos }\end{array}$} & \multirow[b]{2}{*}{$\begin{array}{l}\text { Lower } \\
\text { Bound }\end{array}$} & \multicolumn{2}{|c|}{ Solução Gurobi@ } & \multicolumn{2}{|c|}{ Solução VNS } & \multicolumn{2}{|c|}{ Solução VNDS } \\
\hline & & FO & $\begin{array}{l}\text { GAP } \\
(\%)\end{array}$ & FO & Tempo (s) & FO & Tempo (s) \\
\hline 120 & 93.028 & 97.000 & 2,43 & 97.920 & 218,76 & 97.120 & 207,66 \\
\hline 250 & 194.055 & 204.460 & 4,23 & 204.980 & 957,65 & 204.080 & 909,75 \\
\hline 500 & 385.212 & 431.780 & 8,55 & 407.660 & $7.813,42$ & 407.660 & $7.428,24$ \\
\hline 1.000 & 768.005 & ND & ND & 817.460 & $37.642,67$ & 817.460 & $35.823,00$ \\
\hline
\end{tabular}
Fonte: Autoria própria.

Os resultados na Tabela 2 apresentam os resultados consolidados para os quatro conjuntos de instâncias de testes: o primeiro conjunto possui 20 instâncias de testes com 120 objetos em cada; o segundo conjunto é composto por 20 instâncias de teste com 250 objetos em cada; o terceiro conjunto são 20 instâncias com 500 objetos em cada e 20 instâncias de testes com 1.000 objetos cada compõem o quarto conjunto. Não foi possível encontrar a solução ótima para cada um deles utilizando o Gurobi@ C. Os valores de função objetivo das soluções apresentadas na coluna Solução Gurobi@ na Tabela 2 é a melhor solução obtida pelo pacote de otimização Gurobi@ após uma hora de processamento computacional. Para o conjunto com instâncias com 1.000 objetos, o pacote de otimização Gurobi@ não obteve nenhuma solução. Os resultados da Tabela 2 mostram que a solução obtida pelo Gurobi@ esteve, em média, 7,24\% acima do valor do limite inferior (lower bound), a solução obtida pela meta-heurística VNS esteve, em média, 5,79\% acima e a solução obtida pela meta-heurística VNDS esteve, em média, 5,46\% acima do limite inferior. 
Os resultados obtidos indicam que as meta-heurísticas propostas permitiram obter os melhores resultados para as instâncias com 500 ou 1.000 objetos. E nos demais testes (instâncias com 120 ou 250 objetos) a solução obtida pelas duas meta-heurísticas propostas sempre estiveram próximas da solução obtida pelo Gurobi@) (menos de 1\% acima para instâncias com 120 objetos e menos de 0,3\% acima para instâncias com 250 objetos), o que indica que as mesmas atingem resultados de excelente qualidade com tempos de processamento muito reduzidos. Elas também se mostraram mais rápidas do que a solução do modelo matemático e nos problemas de grande porte (1.000 objetos) o modelo matemático não conseguiu encontrar uma solução. As soluções obtidas pelo Gurobi@ estiveram, em média, entre 4,27\% e 12,09\% acima do limitante inferior (lower bound), a meta-heurística VNS obteve soluções entre 5,26\% e 6,44\% acima do limitante inferior e a meta-heurística VNDS obtive soluções entre 4,40\% e 6,44\% acima do limitante inferior. A meta-heurística VNDS foi entre $4,85 \%$ e 5,10\% mais rápida do que a meta-heurística VNS. Este resultado comprova que as meta-heurísticas conseguem obter soluções com valores mais próximos do limitante inferior do que as soluções obtidas pelo Gurobi@ e possivelmente podem obter soluções próximas da solução ótima.

\title{
Conclusão
}

Inúmeras são as possíveis extensões para a continuidade desta pesquisa, tendo em vista o número reduzido de trabalhos na literatura, incluindo a investigação de novos métodos de solução, por exemplo, outras meta-heurísticas ou modificações na meta-heurística VNDS. A aplicação e validação dos métodos propostos a problemas reais que envolvem o agrupamento de cargas no contexto da distribuição física de produtos também é uma extensão possível, dado que neste trabalho foram utilizados dados de instâncias benchmarking da literatura.

Outra proposta para a continuidade deste trabalho é o estudo de novas estruturas de vizinhança para o problema de binpacking bidimensional (volume e peso dos objetos) com frota heterogênea. Inclusive com novas instâncias de testes com variações nas capacidades (tanto peso, quanto volume) dos veículos e limitações nos tamanhos de frota disponível.

\section{Agradecimentos}

Ao Conselho Nacional de Desenvolvimento Científico e Tecnológico - CNPq - Brasil pelo apoio para a realização da pesquisa que resultou no presente trabalho.

\section{Referências}

Bukard, R. E., \& Zhang, G. (1997). Bounded space on-line variable-sized bin packing. Acta Cybernetica, 13, 63-76.

Castro Júnior, N. (2013). Mensuração de externalidades do transporte de carga brasileiro, Journal of Transport Literature, 7(1), $163-181$.

Coffman, E. G., Garey, M. R., \& Johnson, D. S. (1997). Approximation algorithm for bin-packing: a survey. In: Hochbaum D (eds) Approximation algorithm for NP-hard problems. PWS Publishing, Boston, 46-93.

Correia, I., Gouveia, L. , \& Saldanha-da-gama, F. (2008). Solving the variable size bin packing problem with discretized formulations. Computers \& Operations Research, 35, 2103-2113.

Cunha, C. B, Manieri, G., Yoshizaki, H. T. Y., Maluta, L. , \& Henriques, L. R. S. (2008). Heurísticas para o Problema de Bin-Packing no Contexto da Distribuição Física de Produtos. In: XL SBPO - 40 Simpósio Brasileiro de Pesquisa Operacional, João Pessoa. Anais, 712-723.

Eller, R. A. G., Sousa Junior, W. C. , \& Curi, M. L. C. (2011). Custos do transporte de carga no Brasil: rodoviário versus ferroviário. Journal of Transport Literature, 5(1), 50-64.

Falkenauer E. (1996). A Hybrid Grouping Genetic Algorithm for Bin Packing. Journal of Heuristics, 2(1), 5-30.

Friesen, D. K. , \& Langston, M. A. (1986). Variable-sized bin packing. SIAM J. Comput. 15, 222-230.

Garey, M. R. , \& Johnson, D. S. (1979). Computers and intractability: a guide to the theory of NP-completeness. W. H. Freeman, San Francisco.

Glover, F., \& Kochenberg, G. A. (2003). Handbook of Metaheuristics. International Series in Operations Research \& Management Series, Kluwer's International Series, Stanford University.

Haouari, M., \& Seriari, M. (2009). Heuristics, for the variable sized bin-packing problem, Computers and Operations Research, 36, 28772884.

Hansen, P., Mladenovic, N., \& Perez-brito, D. (2001). Variable neighborhood decomposition search. Journal of Heuristics, 7(4), 335-350.

Mladenovic, N., \& Hansen, P. (1997). Variable neighborhood Search. Computers and Operations Research, 24 (11), 1097-1100.

Miura, M. (2008). Modelagem heurística no problema de distribuição de cargas fracionadas de cimento. Dissertação (Mestrado em Engenharia de Transporte). ed. São Paulo: Universidade de São Paulo.

Reis, J. A., \& Cunha, C. B. (2011). Uma nova Heurística Baseada na Meta-heurística VNS para o Problema de Agrupamento de Entregas em Veículos de uma Frota Heterogênea. Transportes (Rio de Janeiro), 1, 1-18.

Zhang, G. (1997). A New Version of On-line Variable-sized Bin-packing, Discrete Applied Mathematics, 72, 193-197.

\begin{abstract}
In this paper, we deal with the physical distribution problem that comprises grouping and assigning deliveries to a heterogeneous fleet of vehicles aiming to minimize the total freight cost. This problem arises in several practical situations in which the customers to be served are geographically close enough to each other such that distances traveled between consecutive stops can be regarded as irrelevant when compared to the overall vehicle costs, and thus not considered when establishing the freight rate to be paid to third-party carriers. This work proposes a generalized bin-packing problem resolution method with additional constraint found in logistics and freight transportation practical situations. A VNDS (Variable Neighborhood Decomposition Search) meta-heuristic proposed to solve this problem. Computational experiments conducted using benchmark instances problems from the literature evidence that our heuristic produces improved results (optimal solution or near lower limit in all instances) in very short times (5.10\% faster than the VNS).
\end{abstract}

Key words: variable neighborhood decomposition search, bi-dimensional variable sized bin-packing problem, meta-heuristic. 